\title{
Nucleic Acid Aptamers as Molecular Tags for Omics Analyses Involving Sequencing
}

\author{
Masayasu Kuwahara ${ }^{1}$ and Naoki Sugimoto ${ }^{2}$ \\ ${ }^{1}$ Chemistry Laboratory of Artificial Biomolecules (CLAB), \\ Graduate School of Engineering, Gunma University \\ ${ }^{2}$ Frontier Institute for Biomolecular Engineering Research (FIBER) and \\ Faculty of Frontiers of Innovative Research in Science and Technology (FIRST), \\ Konan University \\ Japan
}

\section{Introduction}

Since the beginning of the $\$ 1,000$ genome project, sequencing technologies have progressed rapidly and equipments capable of massively parallel sequencing have been developed. These machines can perform 3G DNA sequencing corresponding to the entire human genome within only a few hours. Indeed, using such machines, quantitative analysis methods for multiple types of RNA, even in a single cell, are very close to being developed. This would be useful for transcriptome studies including mRNA, rRNA, tRNA, and other non-coding RNA. However, these methods would not be applicable to proteome and other omics analyses because non-nucleic acid molecules, such as proteins and peptides, do not have nucleotide sequences. If non-nucleic acid molecules could be converted to a nucleotide sequence, analytical methods that use massively parallel sequencing could also be applied to omics analyses for non-nucleic acid molecules. Nucleic acid aptamers (specific binders) can be readily amplified by polymerase reaction and decoded by sequencing. Therefore, it is possible to apply them as molecular tags to quantitative biomolecular analyses and single cell analyses, among others. This chapter focuses on the development of nucleic acid aptamers and the outlook for related technologies.

\section{Construction of random screening systems}

Random screening refers to a methodology for obtaining molecules that perform a desired activity, such as catalysis and specific binding, by displaying masses of diverse molecules called a library and selecting from them. This method involves phage display [Smith \& Petrenko, 1997] and split-mix combichem [Geysen et al., 2003] as well as in vitro selection [Robertson \& Joyce, 1990; Ellington \& Szostak, 1990; Tuerk \& Gold, 1990], i.e., it is a random screening method for functional nucleic acids.

\subsection{Beginning of screening functional nucleic acids}

Functions of nucleic acids, such as DNA and RNA, have been considered to be only to preserve, transfer, and express genetic information. The prevailing notion is that proteins are 
functional molecules that play an important role in the body and that DNA is the blueprint of proteins and RNA is the mediator. Therefore, the potential of nucleic acids to behave as functional molecules has not attracted much interest. However, since the 1980s, with the discovery by Cech et al. and Altman et al. of RNA enzymes (ribozymes) that catalyze RNA self-cleavage or RNA transesterification in splicing [Zaug \& Cech, 1986; Guerrier-Takada et al., 1983], much interest has been focused on the creation and application of functional nucleic acids with new activities that are different from the typical activities of nucleic acids.

A screening methodology to select functional RNA molecules that can catalyze a specific reaction (ribozyme) or bind to a specific molecule (aptamer) from the RNA library (RNA pool of miscellaneous random sequences) was independently reported by Szostak et al., Joyce et al., and Gold et al. around 1990. This random screening method for functional nucleic acids is called in vitro selection. This is often referred to as SELEX (systematic evolution of ligands by exponential enrichment), particularly when used for screening aptamers. Using the in vitro selection method, successful screenings of functional RNA were demonstrated, followed by the creation of the DNA enzyme as a catalyst and the DNA aptamer as a specific binder [Breaker \& Joyce, 1994].

\subsection{Random screening of nucleic acid aptamers}

Single-stranded RNA and DNA (ssRNA and ssDNA, respectively) with a particular sequence, which can be selected by the SELEX method, exert the aptamer activities by forming a specific steric structure with intramolecular hydrogen bonding, stacking interactions, electrostatic interactions, and metal coordination. Using the SELEX method, various RNA/DNA aptamers, which specifically bind to a broad spectrum of targets involving small molecules such as nucleotides or amino acids, vitamins, and antibiotics; macromolecules such as thrombin; and particles such as Rous sarcoma virus (RSV), have been created artificially [Uphoff et al., 1996]. Since the discovery of the riboswitch early in the $21^{\text {st }}$ century, it has been known that nucleic acid aptamers also exist in nature [Winkler et al., 2002].

In the SELEX method, the screening of nucleic acid aptamer is performed using an ssDNA pool of miscellaneous random sequences as the initial library. Oligonucleotide containing approximately 1013-15 diversities of sequences is chemically synthesized. Typically, its length is approximately forty mer to one hundred and several tens mer, though the length can vary depending on the size of the target and the screening system that the researchers want to construct. In the case of DNA aptamer screening (Fig. 1), the initial library is applied to solid support, such as sepharose gel and nitrocellulose membrane, such that the target is immobilized and active species are separated from nonactive ones. The DNA with target activity is amplified by polymerase chain reaction (PCR) to give the corresponding dsDNA, and then ssDNA is prepared by an appropriate treatment of the complementary strand, such as degradation using $\lambda$ exonuclease and biotin trapping using avidin gels. The resulting ssDNA library is used for the next round, and repeated cycles of separation and amplification lead to enriched sequences that show affinity to the target. The DNA aptamers are individually isolated from the enriched pool using a cloning method, and their sequences and binding properties to the target are analyzed. In the case of RNA aptamer screening, two processes are involved. The initial synthetic DNA library is converted to a RNA library using RNA polymerase and after affinity separation, the active RNA is converted and amplified to DNA using a reverse transcription PCR (RT-PCR); this library is subjected to the previously described DNA aptamer screening scheme. 


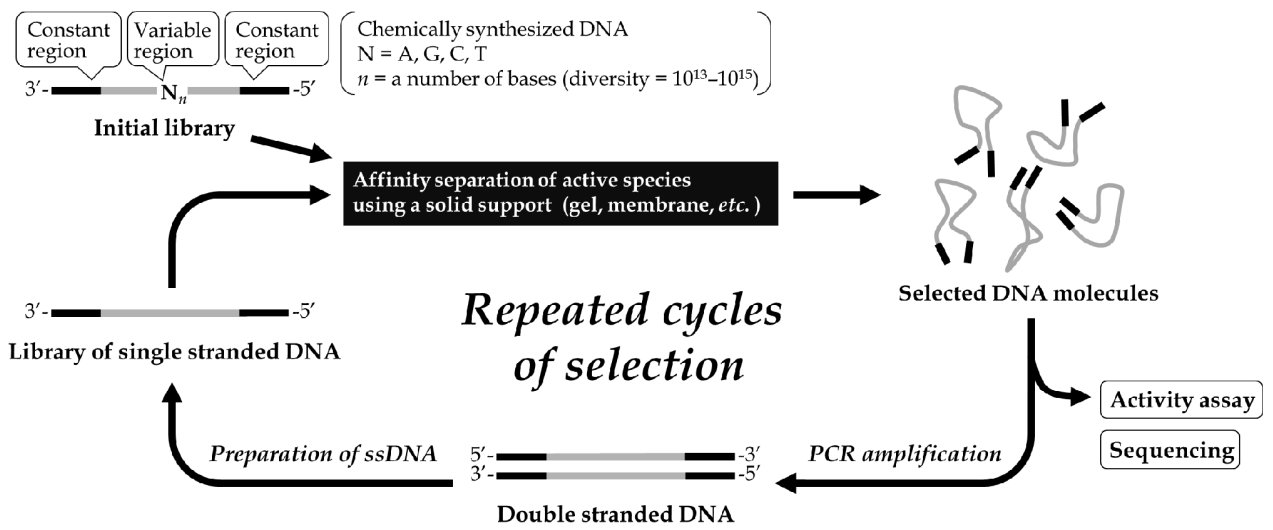

Fig. 1. General scheme of the SELEX method using the DNA library.

\section{Chemical modification}

Many examples of nucleic acid aptamers have been reported. However, some defects have been revealed. A major defect is physical instability due to degradation by endogenous nucleases. Unmodified nucleic acid aptamers are immediately degraded in vivo. Furthermore, a limitation on the binding affinity and specificity are anticipated because nucleic acids consist of a combination of only four nucleotides whereas proteins consist of a combination of twenty amino acids with diverse functional groups in their side chains. Therefore, the development of an advanced screening method to obtain modified nucleic acid aptamers from chemically modified libraries, in which different functionalities that cannot be found in a nucleotide are incorporated, has been performed.

\subsection{Polymerase reaction involving modified nucleotides}

A key step in the SELEX method is the amplification of selected nucleic acid molecules by affinity separation. This method would make good use of a unique feature of the DNA molecule: it can be amplified and replicated through PCR. Other organic molecules do not possess such a feature. In order to adapt the method to modified nucleic acids as well as to natural RNA and DNA, modified nucleic acids should be enzymatically synthesized from a natural DNA template by polymerase reactions with high efficiency. Conversely, DNA should also be efficiently produced from a modified nucleic acid template. Of course, those conversions are not necessary if the modified nucleic acid used could be directly amplified by PCR in the same manner as DNA. 

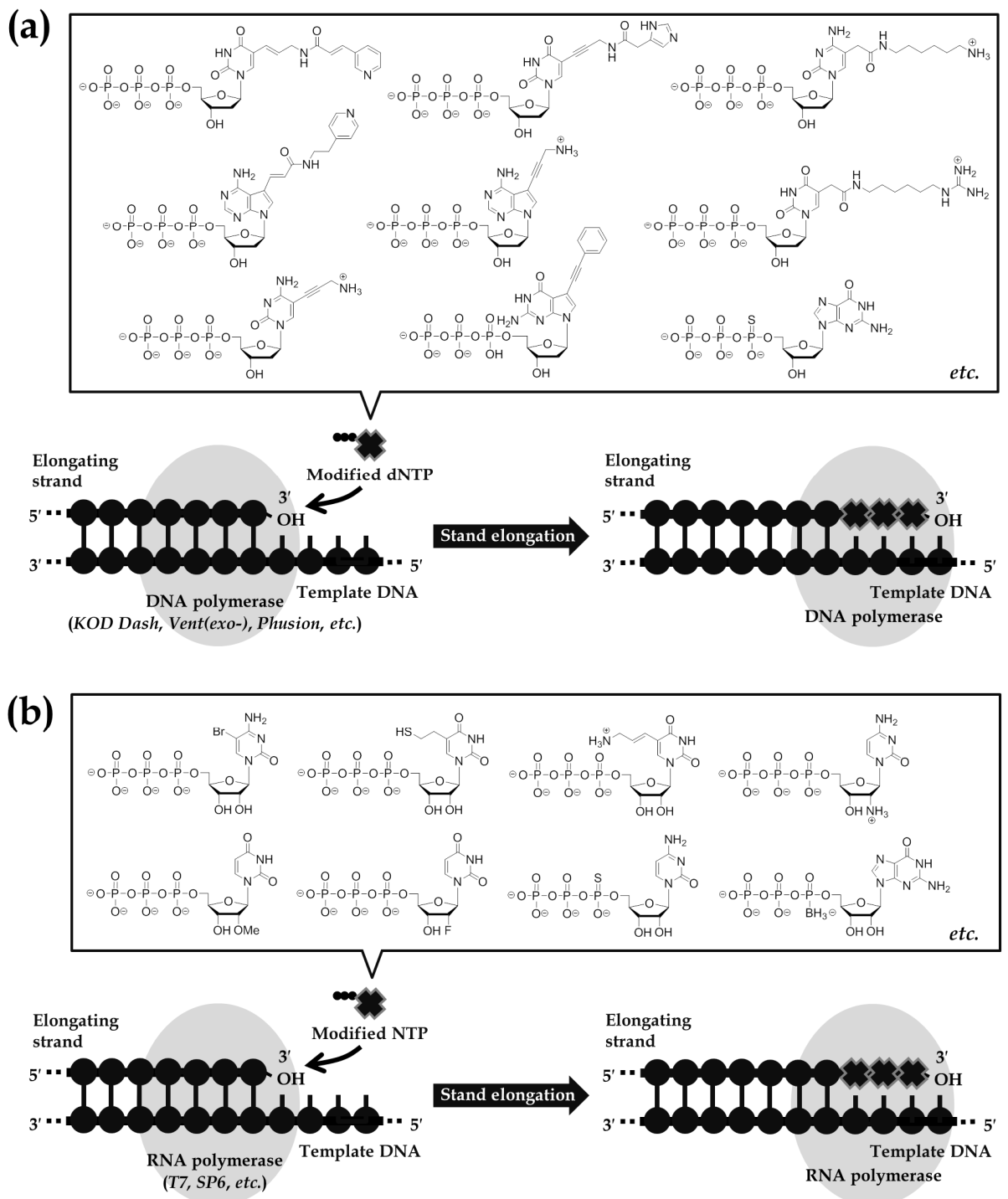

Fig. 2. Enzymatic production of modified DNA (a) and modified RNA (b).

Various types of modified nucleic acids have been designed and polymerase reactions using them have been reported. A monomer unit of nucleic acid consists of a base, sugar, and phosphate moieties, and each component could be an object of chemical modification. When modified nucleic acids are produced from a DNA template, modified nucleoside triphosphate can be used instead of the corresponding natural nucleoside triphosphate as a substrate for the polymerase reaction (Fig. 2a). If the modified nucleoside triphosphate acts 
as a good substrate for the polymerase reaction, the corresponding modified nucleic acids could be efficiently produced. The modification of the sugar and phosphate moieties would tend to decrease the efficiency of the polymerase reaction to a much greater extent than the base moiety does, although this depends on the type of chemical modification [Kuwahara et al., 2006; 2008; 2009]. In particular, 2'-deoxynucleoside-5'-triphosphate analogs with pyrimidine substituted at the 5th position and purine substituted at the 7th or 8th positions of the base moiety tend to be acceptable for DNA polymerase and act as good substrates [Sakthivel \& Barbas, 1998; Lee et al., 2001; Tasara et al., 2003]. Furthermore, $\alpha$-phosphate analogs, where oxygen is replaced with other chemical species, can also be acceptable for DNA polymerase [Andreola et al., 2000]. Analogs with amino $\left(-\mathrm{NH}_{2}\right)$, fluoro (-F), and methoxy (-OMe) groups substituted at the $2^{\prime}$ position of the sugar moiety are known to work in the RNA polymerase reaction [Aurup et al., 1992; Kujau \& Wölfl, 1998].

The chemical structure and replaced position of the modified group as well as the type of polymerase used would affect the production of modified nucleic acids. Among the commercially available thermostable DNA polymerases used for PCR, those belonging to the evolutional family B, such as KOD Dash, Vent(exo-), and Phusion, are thought to be suitable for modified nucleic acid production. In particular, KOD Dash and mutants of KOD DNA polymerases derived from Thermococcus kodakaraensis would be the most suitable for modified DNA synthesis as long as misincorporation rates and successive incorporation of modified substrates are investigated. In contrast, Taq or Tth DNA polymerases, which belong to the evolutional family A, have been found to be sensitive to chemical modification and not suitable for modified DNA synthesis. In PCR, efficient incorporation of modified substrates into the extending strand is required and the modified nucleotide strands produced should act as templates for the next thermal cycle. Though this double barrier make modified DNA synthesis difficult, some 2'-deoxynucleoside-5'-triphosphate analogs of C5-substituted pyrimidine, C7-substituted 7-deaza-purine, and $\alpha$-phosphoro-thioate have been found to be good substrates for PCR when combined with an appropriate DNA polymerase.

Modification of nucleotides often decreases the reaction efficiency and results in small or reduced amounts of the modified DNA with PCR; the modification would cause sequence dependency on the reaction rate, resulting in a bias in the sequences that emerged from the screening. Indeed, kinetic studies on a polymerase reaction using modified nucleotides showed that the reaction with successive incorporations of modified nucleotides was found to be far more inefficient than the reaction with natural nucleotides. In the SELEX using modified DNA, PCR amplification is performed using natural triphosphates in many cases to circumvent this problem. First, the modified DNA is prepared by a primer extension reaction (PEX) using the modified substrate and natural DNA template. After affinity separation, the natural DNA is synthesized and amplified from the selected modified DNA as a template during PCR using natural triphosphates. Then, the modified DNA for the next selection round can be prepared by transcribing the amplified DNA using PEX. This scheme does not contain the simultaneous use of modified triphosphate substrate and modified DNA template. Therefore, the modified DNA, in which many kinds of functionalities are incorporated, can be synthesized more efficiently compared with the previously described method of direct PCR amplification of modified DNA. Indeed, this has enabled SELEX to be used with libraries of doubly or triply modified DNA prepared using PEX [Perrin et al., 2001; Sidorov et al., 2004; Hollenstein et al., 2009]. 
For enzymatic synthesis of modified RNA, the template DNA is transcribed using modified ribonucleoside triphosphates instead of natural triphosphates. Fig. $2 \mathrm{~b}$ shows modified analogs accepted as substrates by RNA polymerase, e.g., T7 RNA polymerase and SP6 RNA polymerase. Modified RNA, particularly that prepared using a uridine analog with various functionalities at the 5th position or uridine/cytidine analogs with a fluorine group or an amino group at the $2^{\prime}$ position, is often applied to the SELEX method. Although the incorporation efficiency of modified analogs is inferior to that of natural substrates, they can provide a relatively long strand of modified RNA as a full-length product corresponding to template DNA. In the SELEX method using modified RNA, after affinity separation, the selected modified RNA is reverse transcribed to DNA and then amplified by PCR; natural nucleoside triphosphates are used as substrates in these processes. The modified RNA for the next selection round is prepared by transcribing the amplified DNA. In reverse transcription, AMV (avian myeloblastosis virus) reverse transcriptase, Tth DNA polymerase with reverse transcription activity, or other types of modified RNA polymerases are often used. The aforementioned modified RNAs were found to act as templates for the reverse transcription catalyzed by these polymerases to yield the corresponding DNA strands.

\subsection{Modified nucleic acid aptamers}

To use nucleic acid aptamers in vivo, chemical modification is indispensable. The first aptamer drug, "Macugen," which is used to treat age-related macular degeneration (AMD), comprises modified RNA including 2'-fluoropyrimidine nucleotides (U, C) and 2'-methoxy purine nucleotides (A, G). This aptamer was created as follows: 1) Sequences that bound to vascular endothelial growth factor (VEGF) using a modified RNA library including 2'-fluoropyrimidine nucleotides were selected by SELEX; 2) Natural purine nucleotides were replaced with 2'methoxy purine nucleotides, in part, only at sites where its binding affinity was retained after the replacement; 3) Chemical modifications of the 5' and 3' ends with branched polyethylene glycol strands and 3'-thymidylate were introduced [Ruckman et al., 1998].

In step 1 of the aforementioned process, T7 RNA polymerase was used as an enzyme, and 2'-fluoropyrimidine nucleoside triphosphates and natural purine nucleoside triphosphates were used as substrates to prepare the modified RNA library. Here, modified purine nucleoside triphosphates could not be used due to the limited capability of T7 RNA polymerase in producing modified RNA. Steps 2 and 3 are called post-SELEX chemical modifications, which can increase nuclease resistance and extend the periods of hemodynamic stasis. In general, chemical modifications have been shown to remarkably enhance biostability and occasionally increase the binding capability of nucleic acid aptamers, as in the following examples.

A sugar-modified RNA aptamer specific to human neutrophil elastase (HNE) is highly stable in serum with a half-life of approximately 20 hours, while natural RNA is degraded within approximately 5 minutes [Lin et al., 1994]. This aptamer includes 2'-aminopyrimidine nucleotides (U, C) instead of the corresponding natural nucleotides. In addition, the improved nuclease resistance of modified RNA aptamers with other types of modifications, i.e., 2'-fluorine and 2'-methoxy groups, were experimentally confirmed. Furthermore, inserting only a single bridged nucleotide analog having $2^{\prime}-\mathrm{CH}(\mathrm{Ph}) \mathrm{OCH}_{2}-4$ ' at the $3^{\prime}$ end of a thrombin binding DNA aptamer (TBA) could increase the resistance to venom exonuclease up to approximately 30 times (Fig. 3) [Kasahara et al., 2010]. 

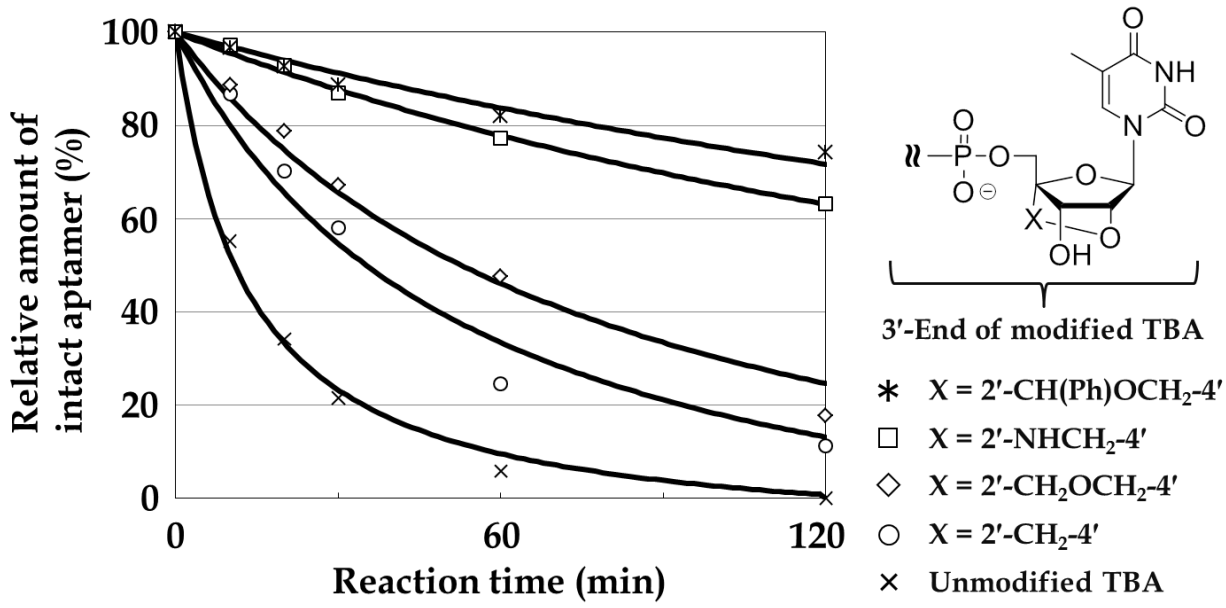

Fig. 3. The time course of the degradation of thrombin binding DNA aptamers (TBAs) by phosphodiesterase I; Total quantities of the products were set at $100 \%$ in each reaction mixture.

A base-modified RNA aptamer specific to the human immunodeficiency virus (HIV) Rev protein obtained from a library of modified RNA containing 5-iodouridine instead of uridine could be bound to the target with a somewhat higher binding affinity $\left(\mathrm{K}_{\mathrm{d}}=0.8 \mathrm{nM}\right)$ compared with the corresponding natural RNA aptamer [Jensen et al., 1995]. The unique feature of this base-modified aptamer is to form a cross-link with the target protein by UV irradiation; halogenated uracil is known to form a covalent bond with a nearby electron-rich amino acid residue by photoirradiation.

Such photo-cross-linkable aptamers are called photoaptamers. They are generally created by photoSELEX, which involves a photo-cross-linking process in the conventional selection cycle. Photoaptamers, in particular, would have an advantage for use in protein assays because much more stringent washing of excess or non-specific proteins can be done compared with antibody-based sandwich assays, because photoaptamers can covalently and irreversibly bind to a target.

A few phosphate-modified RNA aptamers have been reported because the commonly used T7 RNA polymerase could accept limited triphosphate analogs modified at the phosphate moiety as substrates. Modified nucleoside triphosphates involving phosphorothioate and boranophosphate [Lato et al., 2002] are normally used as substrates. A phosphate-modified RNA aptamer specific for bFGF was obtained by screening the library of modified RNA exclusively involving phosphorothioate inter-nucleoside linkages, which could greatly enhance nuclease resistance [Jhaveri et al., 1998]. The aptamer bound to bFGF with a binding affinity of $K_{d}=1.8 \mathrm{nM}$ which was approximately five times lower than that observed for a 2'amino RNA aptamer for the same target $\left(\mathrm{K}_{\mathrm{d}}=0.35 \mathrm{nM}\right)$. However, its strong nuclease resistance was confirmed.

Similarly, various modified DNA aptamers involving 2'-deoxynucleoside analogs with base or phosphate modifications have been selected by SELEX using modified DNA libraries. In both cases with modified RNA/DNA, the inefficiency of enzymatically modified nucleic acid production would occasionally become a bottleneck. Another methodological approach 
to create modified nucleic acid aptamers is a mirror-image aptamer coined Spiegelmer. To obtain Spiegelmers, there is no need to enzymatically prepare modified nucleic acid libraries during the selection cycles. Instead, screening is performed using natural RNA/DNA libraries by means of a mirror image of the target molecule. After screening, mirror-image aptamers with the same sequences as the selected aptamers were chemically synthesized using L-ribonucleotides or L-deoxyribonucleotides. Until date, Spiegelmers that bind specifically to L-arginine, D-adenosine, L-vasopressin, and others have been created; they were found to have improved nuclease resistance [Nolte et al., 1996].

\section{Affinity and specificity}

Affinities of well-known representative nucleic acid aptamers are shown in Fig. 4, along with those of specific protein binders, such as antibody, streptavidin, and rectin. In general, the binding affinity between molecules can be numerically expressed by the dissociation constant $\left(K_{d}\right)$. At equilibrium for the association/dissociation reaction $R+L \rightleftarrows R L, K_{d}$ is defined by: $K_{d}=[R][L] /[R L]$, where $[R],[L]$, and [RL] are concentrations of free receptor, free ligand, and their interaction complex, respectively; smaller $\mathrm{K}_{\mathrm{d}}$ values indicate higher binding affinities. For example, $\mathrm{K}_{\mathrm{d}}$ values of biotin/streptavidin and digoxigenin/antidigoxigenin antibody, which are often used as research reagents, are $4 \times 10^{-14}(40 \mathrm{fM})$ and $1.1 \times 10^{-8}(11 \mathrm{nM})$, respectively [Holmberg et al., 2005; Tetin et al., 2002]. Those for galactose/jacalin and mannose/concanavalin A are $1.6 \times 10^{-5}(16 \mu \mathrm{M})$ and $2.0 \times 10^{-4}(200$ $\mu \mathrm{M})$, respectively [Smith et al., 2003]. Immunoglobulin $\mathrm{G}(\mathrm{IgG})$ has $\mathrm{K}_{\mathrm{d}}$ values of approximately $10^{-7}(100 \mathrm{nM})$ or less, which is classified as a high affinity antibody.

$\mathrm{K}_{\mathrm{d}}$ values of protein binding aptamers specific for keratinocyte growth factor (KGF), vascular endothelial growth factor (VEGF), and thrombin are $3 \times 10^{-13}(0.3 \mathrm{pM}), 5 \times 10^{-11}(50 \mathrm{pM})$, and 5 $\times 10^{-10}(500 \mathrm{pM})$, respectively; these have excellent binding affinities [Pagratis et al., 1997; Kubik et al., 1994; Ruckman et al., 1998]. The VEGF binding aptamer is Macugen. The $K_{d}$ value of an anti-VEGF antibody, bevacizumab, which is used for cancer therapy, is $1.1 \times 10^{-9}(1.1$ $\mathrm{nM})$. In general, the $\mathrm{K}_{\mathrm{d}}$ value required for antibody drugs for molecular targeted therapies should be approximately $10^{-9}(1 \mathrm{nM})$ or less. Hence, nucleic acid aptamers comparable to antibodies in terms of binding affinity can be created by SELEX with sophisticated techniques as long as the target is a high molecular weight molecule like a protein.

Regarding nucleic acid aptamers specific for small molecules, the $\mathrm{K}_{\mathrm{d}}$ value of a biotin binding aptamer, for example, is about $6 \times 10^{-6}(6 \mu \mathrm{M})$, which is $10^{8}$ or more times greater than that of streptavidin [Wilson et al., 1998]. Except for a few examples, the reported small molecule binding aptamers that have been artificially created have $K_{d}$ values ranging from approximately $10^{-7}$ to $10^{-4}$ (several hundred $\mathrm{nM}$ to several hundred $\mu \mathrm{M}$ ). To use these as research reagents and diagnostic agents, their binding affinities need to be increased up to at least $10^{-7}$ or less, hopefully $10^{-8}$ or less.

Ligand binding sites of riboswitches found in the mRNA of bacteria and the like are considered to be naturally occurring aptamers. Some of these aptamers exhibit very high binding affinities for their small molecule targets, and their $\mathrm{K}_{\mathrm{d}}$ values are several $\mathrm{nM}$. For example, $\mathrm{K}_{\mathrm{d}}$ values of aptamers specific for S-adenosyl-methionine, flavin mono nucleoside, and guanine are $4 \times 10^{-9}(4 \mathrm{nM}), 5 \times 10^{-9}(5 \mathrm{nM})$, and $5 \times 10^{-9}(5 \mathrm{nM})$, respectively [Winkler et al., 2003; Winkler et al., 2002; Mandal et al., 2003]. Thus, it is possible that aptamers that bind strongly to small molecules could be created by further improvements in screening methods. 


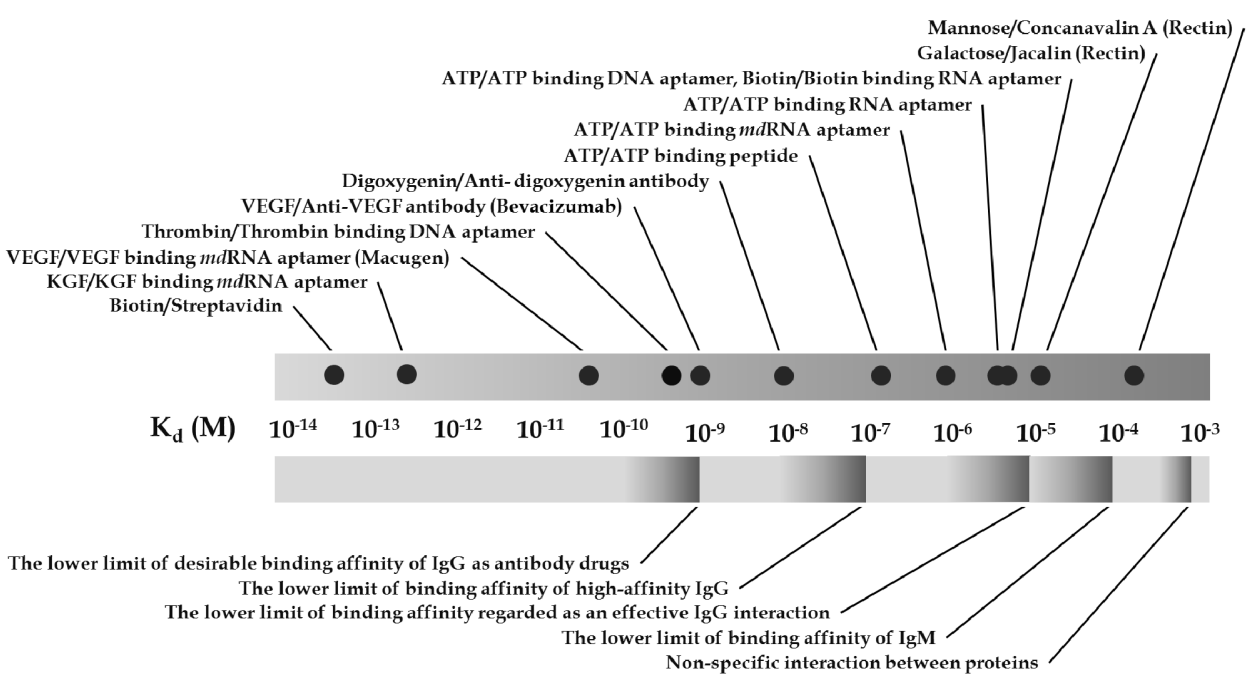

Fig. 4. Comparisons of binding affinities of well-known representative nucleic acid aptamers with those of proteins having specific binding abilities.

Effects of chemical modifications on binding affinity and specificity remain unclear till date. However, it has been suggested that chemical modifications that could constrain structural fluctuations would favorably influence binding affinity. This does not contradict that, in many cases, a target/nucleic acid aptamer complex can be stabilized with divalent metal cations like $\mathrm{Mg}^{2+}$ and $\mathrm{Ca}^{2+}$. To characterize effects of chemical species in a library in terms of affinity and specificity, binding properties of aptamers specific for a target, which are respectively selected from libraries different in chemical species using a standardized common screening protocol, should be systematically analyzed.

In previous studies, ATP binding aptamers were obtained respectively from RNA, DNA, and modified RNA and modified DNA, although there were some differences in the screening protocol [Sazani et al., 2004; Huizenga \& Szostak, 1995; Vaish et al., 2003; Battersby et al., 1999]. Modified RNA and modified DNA contain 5-(3-amino)propyl-uridine and 5-(3amino)propyl-2'-deoxyuridine, respectively. $K_{d}$ values of the obtained aptamers ranged from approximately $10^{-7}$ to $10^{-6}$ (several hundred $\mathrm{nM}$ to several $\mu \mathrm{M}$ ), and remarkable differences depending on chemical species in libraries were not observed. Incidentally, the $\mathrm{K}_{\mathrm{d}}$ value of an ATP binding peptide comprised 62 amino acids obtained using mRNA display selection was $1.9 \times 10^{-7}(190 \mathrm{nM})$; the binding affinity of this peptide is likely to be higher to some degree than those of the aforementioned nucleic acid aptamers [Chaput \& Szostak, 2004].

Fig. 5 shows a comparison of the binding specificities of an RNA aptamer, a modified RNA aptamer, and a peptide that bind to ATP. The affinity of the RNA aptamer is 64-fold lower because of a lack of $\gamma$-phosphate in ATP, and is 1100-fold lower because of lack of both $\beta$ - and $\gamma$-phosphates. Although its affinity is 600 -fold lower because of a replacement of an adenine base with a pyrimidine base, its specificities for the base and sugar moieties in ATP tend to be less sensitive than that for the phosphate moiety. In contrast, the modified RNA 
aptamer accurately recognizes the base moiety, but its specificities for phosphate and sugar moieties are likely to be low. Base discernment of the ATP binding peptide is very high; it can accurately distinguish even hypoxanthine in ITP from adenine in ATP. It can recognize the sugar moiety much more clearly than the other two binding molecules, but it is inferior to the RNA aptamer with regard to recognizing the phosphate moiety. While the ATP binding peptide would recognize the target over its entire structure, the ATP binding nucleic acid aptamers also show high recognition capabilities, such as discriminating the detailed partial structures of the target molecule. Unfortunately, comparisons of RNA and modified RNA aptamers do not indicate any superiority owing to the introduction of chemically modified groups. However, (3-amino) propyl groups introduced were found to be essential in order that the modified RNA aptamer could bind to ATP, which indicated that the modified RNA aptamer could recognize the target molecule in a manner that was different from that of a natural RNA aptamer.
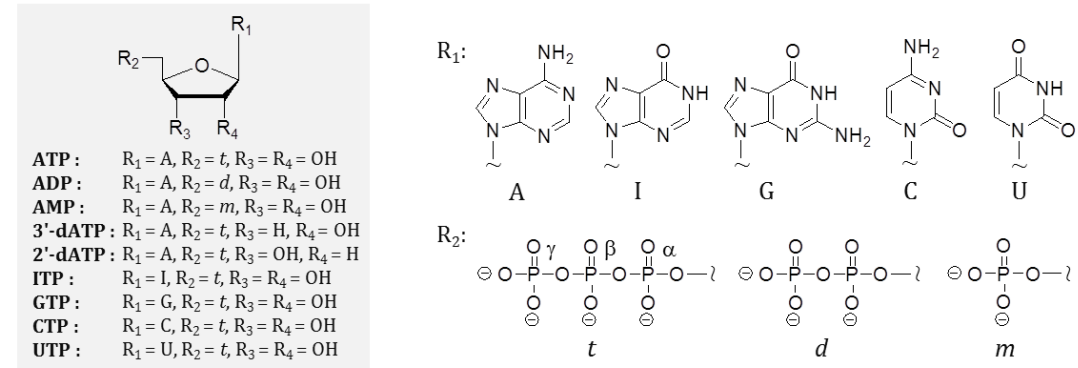

\begin{tabular}{|c|c|c|c|c|c|c|c|c|c|}
\hline Recognition site & \multicolumn{3}{|c|}{ Phosphate moiety } & \multicolumn{2}{|c|}{ Sugar moiety } & \multicolumn{4}{c|}{ Base moiety } \\
\hline Target molecule & ATP & ADP & AMP & 3'-dATP & 2 -dATP & ITP & GTP & CTP & UTP \\
\hline ATP binding RNA aptamer & 1 & 64 & 1100 & 8.5 & 10 & 66 & 19 & 680 & 610 \\
\hline ATP binding $m d$ RNA aptamer & 1 & 1 & 2.1 & 2.5 & 2.4 & 150 & NB & NB & NB \\
\hline ATP binding peptide & 1 & 6.8 & 17 & 40 & 190 & $>5000$ & $>5000$ & $>5000$ & $>5000$ \\
\hline
\end{tabular}

Fig. 5. Comparisons of binding specificities of an RNA aptamer, a $m d R N A$ aptamer, and a peptide that bind to adenosine-5'-triphosphate (ATP).

Similarly, a modified DNA aptamer that can enantioselectively recognize an R-isomer of a thalidomide analog, loses its binding activity $\left(\mathrm{K}_{\mathrm{d}}=1 \times 10^{-6} \mathrm{M}\right)$ if aminohexyl-carbamoylmethyl groups, introduced as a foreign functionality, are removed; i.e., the modified uracil bases are replaced with natural thymine bases [Shoji et al., 2007]. Interestingly, despite the high enantioselectivity of this aptamer, the binding affinity did not decrease if the hydrogen group at the asymmetric carbon was replaced with a methyl group. According to a model that simulated a stable conformation and that was confirmed by experimental results, the hydrogen group projected outward into the water solution. The thalidomide analog is stacked with an adenine base, and the foreign amide group forms a hydrogen-bonding network together with the thalidomide analog and neighboring base, which provides stability to the complex. Thus, the chirality of such a highly symmetric low-molecular-weight compound could clearly be recognized with the assistance of the modified groups that were introduced. 


\section{Development of advanced screening system}

Although the SELEX method has been successfully used to create nucleic acid aptamers, further improvements are required to make the screening process much more rapid and convenient. Nucleic acid aptamers that show high activities in vitro are not always as effective in vivo. Alternative evolutional approaches to create nucleic acid aptamers that can function in living cells and organisms are also being developed.

\subsection{Capillary electrophoresis-SELEX}

Capillary electrophoresis (CE)-SELEX and associated improved methods have been developed to obtain protein binding nucleic acid aptamers quickly and easily (Fig. 6a) [Mendonsa \& Bowser, 2005]. The common feature of these methods is the use of CE in the affinity separation process, whereas solid supports, such as sepharose gel and nitrocellulose membranes, are used for conventional SELEX methods. Association and dissociation reactions between the target and nucleic acids occur in solution; therefore, enrichment of undesired sequences that bind to the solid support would be avoidable. In addition, use of an automated $\mathrm{CE}$ device with high resolving power can greatly reduce the number of repeated cycles of the screening operation. Conventional SELEX typically requires 8-15 selection cycles. CE-SELEX can complete the process in only 2-4 rounds. To date, various DNA aptamers that bind to the human immunodeficiency virus transferase (HIV-RT), immunoglobulin E (IgE), mismatch repair protein (MutS), and ricin, among others, have been obtained by CE-SELEX methods. These resulting DNA aptamers possess a sufficient binding ability as a specific binder. Their $\mathrm{K}_{\mathrm{d}}$ values are $0.18 \mathrm{nM}, 23 \mathrm{nM}, 3.6 \mathrm{nM}$, and $58 \mathrm{nM}$, respectively [Mosing et al., 2005; Mendonsa \& Bowser, 2004; Drabovich et al., 2005; Tang et al., 2006; ].

For CE-SELEX, an uncoated fused-silica capillary can be used because both nucleic acid and the inner wall of the capillary are negatively charged during electrophoresis. Capillaries in which the inner wall is coated are often used for CE separation analyses of biomolecules to suppress peak tailing due to interaction between analytes and the inner wall. The length of the oligonucleotide for the initial library is about 30 to 40 mer including 70 to 90 mer of the random sequence region. In many cases, the 5 '-end of the oligonucleotide is labeled with a fluorophore, such as fluorescein (FAM), to detect the active species in the library using a laser induced fluorescence (LIF) detector, which is far more sensitive than a UV-absorbance detector.

In a typical CE-SELEX method of protein binding DNA aptamers, the library is incubated with the target. The mixture is injected into a capillary and subsequently separated using nonequilibrium CE of equilibrium mixtures (NECEEM). The sample and detector are set on the cathode and anode side, respectively, because electroosmotic flow (EOF) toward anode from cathode is generated when voltage is applied (Fig. 6b). Small and positively charged substances would migrate faster in the capillary. In many cases, DNA that forms a complex with the target protein would reach the detector prior to unbound free DNA, resulting in a clear separation between active and inactive species. However, if the molecular weight of the target protein is considerably large, at times, peaks of bound DNA and unbound DNA get closer and cannot be separated enough to conduct the screening. In such cases, the capillary with which the inner wall is coated, e.g., polyvinyl alcohol is used for the 
separation. Analytes migrate toward the cathode from the anode in the running buffer at a $\mathrm{pH}$ higher than the isoelectric point ( $\mathrm{pI}$ ) of the target protein because EOF does not generate in the coated capillary. Therefore, contrary to the case using a fused-silica capillary, the sample and detector are set on the anode side and the cathode side, respectively.
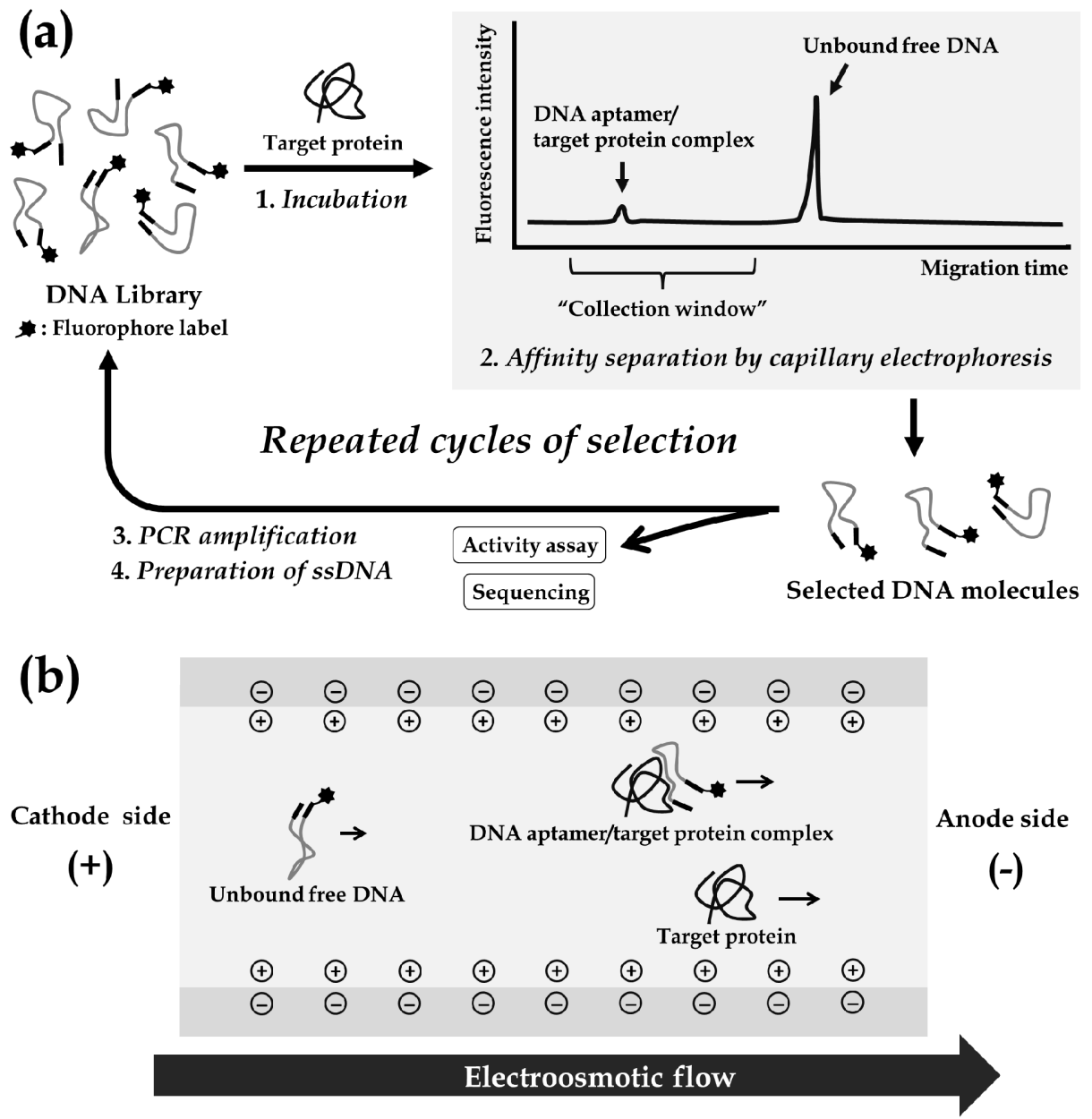

Fig. 6. General scheme of the CE-SELEX method (a); Affinity separation of the bund DNA from the unbound DNA using free zone CE (b).

During electrophoresis, association and dissociation between DNA and target protein is in a non-equilibrium state, and DNA gradually dissociates from the target protein. Therefore, DNA aptamers with a slow dissociation rate are expected to be obtained if a longer capillary is used. However, the migration time is proportional to the length of the capillary. The injection volume of the sample could be increased if a capillary with a larger inner diameter (i.d.) is used. However, the use of a thick capillary makes the release of Joule heating 
inefficient. Joule heating should be avoided because the active structure of the DNA aptamer would be destabilized due to heat denaturation. Thus, optimizations of capillary length and i.d. are important to obtain the target DNA aptamer efficiently. In many cases, capillaries with a length of approximately $31-80 \mathrm{~cm}$ and an i.d. of 50 or $75 \mu \mathrm{m}$ are used.

Limitation of the library size is a defect of CE-SELEX; only several nanoliters of sample mixture can be injected per affinity separation. In the conventional SELEX method, the initial library normally contains miscellaneous sequences with 1013-15 diversities. If CESELEX were performed using a library of the same size, a sample mixture with a concentration of several tens to several hundreds $\mathrm{mM}$ has to be injected. With such a high DNA concentration, it is very difficult to separate the DNA aptamer/target protein complex from the free DNA by CE. Therefore, for CE-SELEX, the size of initial library is approximately $10^{10-13}$.

Regardless of this limitation, CE-SELEX provides an effective methodology to obtain target DNA aptamers with a high incidence rate in a few selection rounds and there is no requirement of negative selection. For example, in the screening of $\operatorname{IgE}$ binding DNA aptamer by CE-SELEX, most sequences obtained in the enriched pool showed binding activity; almost $100 \%$ enrichment was achieved. Such fast enrichments of active species have not been reported for the conventional SELEX method in the literature; the enrichment has rarely been found to be above $50 \%$ within the first few rounds. Furthermore, non-SELEX selection, with no need to repeat tiresome selection cycles, has been developed as an advanced type of CE-SELEX. Thus, the generation rate for nucleic acid aptamers has dramatically increased, and the process has become more convenient by applying $\mathrm{CE}$, with high resolving power, to the SELEX method.

\subsection{Cell-SELEX}

Cell-SELEX is a methodology used to obtain nucleic acid aptamers that specifically bind to a particular cell line [Daniels et al., 2003]. This method does not require prior knowledge of targets and would, in principle, generate nucleic acid aptamers for multiple targets simultaneously on the cell surface. Until now, nucleic acid aptamers specific to a number of cell lines, including a T-cell line (human acute lymphoblastic leukemia), B-cell line (human Burkitt lymphoma), and mouse liver hepatoma cell line, have been reported [Shangguan et al., 2006; Raddatz et al., 2008; Shangguan et al., 2008]. One problem with the conventional SELEX technique is that undesired sequences bind to the solid support such that the target becomes immobilized, causing negative selection to be performed. In cell-SELEX, sequences bound to the common cell matrix also need to be excluded. Therefore, in many cases, counter selection using a cell line akin to the target cell line is performed before or after selection using the target.

In the screening of cell-specific aptamers, an ssDNA library containing miscellaneous sequences with 1014-15 diversities is incubated with the target cell. Unbound sequences are subsequently removed by washing or centrifugation and bound sequences are amplified by polymerase chain reaction followed by preparation of ssDNA for the next selection round. The cell-specific aptamers can be obtained by repeating these processes approximately 20 times. For the present selection, living cells were used, although the sample was partially contaminated with dead cells. Dead cells are known to strongly and independently absorb 
nucleic acid sequences, interfering with enrichment of the target aptamers. To solve this problem, a method using fluorescence-activated cell sorting (FACS) has been developed [Raddatz et al., 2008]. Use of FACS enables dead cells to be removed and active and inactive species to be separated at the same time.

There are many proteins that cause gene expression and a cellular response through signal transductions on the cell surface. Using nucleic acid aptamers specific to these proteins as targets, development of inhibitors that suppress their actions to achieve cell-specific drug delivery has been attempted. To date, nucleic acid aptamers that specifically bind to the cytokine interleukin-17 receptor and human RET receptor tyrosine kinase, for example, have been created by cell-SELEX, and their specific inhibition of the target proteins has been confirmed experimentally [Chen et al., 2011]. As for examples of drug-delivery systems, conjugations of cell membrane protein binding nucleic acid aptamers to siRNAs, toxic proteins, nanoparticles, and antitumor drugs have been developed [McNamara et al., 2006; Chu et al., 2006]. Among these, modified nucleic acids, e.g., RNAs with 2'-fluoro and 2'amino nucleotides and phosphorothioate DNAs, which possess enhanced biostability, are included.

In the conventional SELEX technique using purified proteins as targets, it is anticipated that the nucleic acid aptamer obtained may not be able to bind to the target on the living cell. In contrast, the cell-SELEX can circumvent this problem because screening is performed using actual living cells. By choosing an appropriate cell for the counter selection, researchers can select nucleic acid aptamers targeting either known or unknown molecules, as well as the matrix structure particular to a certain cell line. Thus, cell-SELEX has provided a viable means to create nucleic acid aptamers with significant potential for in vivo application.

\section{Outlook}

The application range of nucleic acid aptamers can be very broad because of their notable advantages over antibodies. For example, nucleic acid aptamers can be obtained by random screening without using laboratory animals, and they can be produced by organic synthesis at low cost. While further technical innovations are still required to bring the performances of nucleic acid aptamers close to those of antibodies, many applications in bioanalyses ranging from test tube assays such as colorimetric sensing to imaging of living cells and tissues as well as therapeutic drugs have been investigated because of their aforementioned advantages. For in vitro assays, natural nucleic acid aptamers are used in many cases; however, chemical modifications assume increased importance in in cell and in vivo analyses to avoid degradation by nucleases. Therefore, development of rapid/facile screening techniques and in vivo screening techniques for creating modified nucleic acid aptamers will contribute to further advances in current bioanalytical measurements. In omics studies for non-nucleic acid molecules such as proteins, peptides, sugars, metabolites, until date, methodologies based on mass spectrometry are mainstreams. However, DNA molecules can readily be amplified by polymerase reactions such as PCR and rolling circle amplification, which generate $10^{6}-10^{9}$ copies of an original sequence. As observed in a riboswitch, RNA molecules cause dynamic structural conversion by association of such small molecules like metabolites to provide multiple transcripts as a molecular offspring. Technologies involving $10^{8}$ or more parallel sequencing and single molecule sequencing reactions are currently established. Considering these unique features of nucleic acid molecules and recent progress 
of sequencing technologies, aptamer/sequencing based analyses, which enable integrated omics analyses at single-cell level, may be developed, and these may replace the present methods for omics analyses in future.

\section{Acknowledgment}

This work was supported in part by a Grant for Industrial Technology Research from the New Energy and Industrial Technology Development Organization (NEDO) of Japan; Grants-in-Aid for Scientific Research, the "Core Research" project (2009-2014), and the "Academic Frontier" project (2004-2009) from the Ministry of Education, Culture, Sports, Science and Technology, Japan; the Hirao Taro Foundation of the Konan University Association for Academic Research; and the Long-Range Research Initiative (LRI) Project of the Japan Chemical Industry Association.

\section{References}

Andreola, M. L., Calmels, C., Michel, J., Toulmé, J. J. \& Litvak, S. (2000). Towards the selection of phosphorothioate aptamers optimizing in vitro selection steps with phosphorothioate nucleotides, Eur. J. Biochem. 267(16), 5032-5040.

Aurup, H., Williams, D. M. \& Eckstein F. (1992). 2'-Fluoro- and 2'-amino-2'-deoxynucleoside 5'-triphosphates as substrates for T7 RNA polymerase, Biochemistry 31(40): 96369641.

Bagalkot, V., Farokhzad, O. C., Langer, R. \& Jon, S. (2006). An aptamer-doxorubicin physical conjugate as a novel targeted drug-delivery platform, Angew. Chem. Int. Ed., 45(48): 8149-8152.

Bagalkot, V., Zhang, L., Levy-Nissenbaum, E., Jon, S., Kantoff, P. W., Langer, R. \& Farokhzad, O. C. (2007). Quantum dot-aptamer conjugates for synchronous cancer imaging, therapy, and sensing of drug delivery based on bi-fluorescence resonance energy transfer, Nano Lett., 7(10): 3065-3070.

Battersby, T. R., Ang, D. N., Burgstaller, P., Jurczyk, S. C., Bowser, M. T., Buchanan, D. D., Kennedy, R. T. \& Benner, S. A. (1999). Quantitative analysis of receptors for adenosine nucleotides obtained via in vitro selection from a library incorporating a cationic nucleotide analog, J. Am. Chem. Soc., 121(42): 9781-9789.

Berezovski, M., Musheev, M., Drabovich, A. \& Krylov, S. N. (2006). Non-SELEX selection of aptamers, J. Am. Chem. Soc., 128(5): 1410-1411.

Breaker, R. R. \& Joyce, G. F. (1994). A DNA enzyme that cleaves RNA, Chem Biol. 1(4): 223229.

Bock, L. C., Griffin, L. C., Latham, J. A., Vermaas, E. H. \& Toole, J. J. (1992). Selection of single-stranded DNA molecules that bind and inhibit human thrombin, Nature 355(6360): 564-566.

Chaput, J. C. \& Szostak, J. W. (2004). Evolutionary optimization of a nonbiological ATP binding protein for improved folding stability, Chem Biol., 11(6): 865-874.

Chen, L., Li, D. Q., Zhong, J., Wu, X.L., Chen, Q., Peng, H. \& Liu, S. Q. (2011). IL-17RA aptamer-mediated repression of IL-6 inhibits synovium inflammation in a murine model of osteoarthritis, Osteoarthr. Cartil., 19(6): 711-718.

Chu, T.C., Marks, J.W. III, Lavery, L. A., Faulkner, S., Rosenblum, M. G., Ellington, A. D. \& Levy, M. (2006). Aptamer:toxin conjugates that specifically target prostate tumor cells, Cancer Res., 66(12): 5989-5992. 
Daniels, D. A., Chen, H., Hicke, B. J., Swiderek, K. M., Gold, L. (2003). A tenascin-C aptamer identified by tumor cell SELEX: systematic evolution of ligands by exponential enrichment, Proc. Natl. Acad. Sci. U S A, 100(26): 15416-15421.

Drabovich, A., Berezovski, M. \& Krylov, S. N. (2005). Selection of smart aptamers by equilibrium capillary electrophoresis of equilibrium mixtures (ECEEM), J. Am. Chem. Soc., 127(32): 11224-11225.

Ellington, A. E. \& Szostak, J. W. (1990). In vitro Selection of RNA molecules that bind specific ligands, Nature 346(6287): 818-822.

Geysen, H. M., Schoenen, F., Wagner, D. \& Wagner, R. (2003). Combinatorial compound libraries for drug discovery: an ongoing challenge, Nat. Rev. Drug Discov. 2(3): 222 230.

Guerrier-Takada, C., Gardiner, K., Marsh, T., Pace, N. \& Altman, S. (1983). The RNA moiety of ribonuclease $\mathrm{P}$ is the catalytic subunit of the enzyme, Cell 35(3): 849-857.

Hollenstein, M., Hipolito, C. J., Lam, C. H. \& Perrin, D. M. (2009). A self-cleaving DNA enzyme modified with amines, guanidines and imidazoles operates independently of divalent metal cations $\left(\mathrm{M}^{2+}\right)$. Nucleic Acids Res. 37(5): 1638-1649.

Holmberg, A., Blomstergren, A, Nord, O., Lukacs, J. Lundeberg, J. \& Uhlén, M. (2005). The biotin-streptavidin interaction can be reversibly broken using water at elevated temperatures, Electrophoresis, 26(3): 501-510.

Huizenga, D. E., \& Szostak, J. W. (1995). A DNA aptamer that binds adenosine and ATP, Biochemistry, 34(2): 656-665.

Jensen, K. B., Atkinson, B. L., Willis, M. C., Koch, T. H. \& Gold, L. (1995). Using in vitro selection to direct the covalent attachment of HIV-1 Rev protein to high affinity RNA ligands. Proc. Natl. Acad. Sci. USA 92(26): 12220-12224.

Jhaveri, S., Olwin, B. \& Ellington, A.D. (1998). In vitro selection of phosphorothiolated aptamers. Bioorg. Med. Chem. Lett. 8(17): 2285-2290.

Kasahara, Y., Kitadume, S., Morihiro, K., Kuwahara, M., Ozaki, H., Sawai, H., Imanishi T. \& Obika S. (2010). Effect of 3'-end capping of aptamer with various 2',4'-bridged nucleotides: Enzymatic post-modification toward a practical use of polyclonal aptamers, Bioorg. Med. Chem. Lett. 20(5): 1626-1629.

Klussmann, S., Nolte, A., Bald, R., Erdmann, V. A. \& Furste, J. P. (1996). Mirror-image RNA that binds D-adenosine, Nat. Biotechnol. 14(9): 1112-1115.

Kubik, M. F., Stephens, A. W., Schneider, D., Marlar, R. A. \& Tasset, D. (1994). High-affinity RNA ligands to human $\alpha$-thrombin. Nucleic Acids Res., 22(13): 2619-2626.

Kujau, M. J. \& Wölfl, S. (1998). Intramolecular derivatization of 2'-amino-pyrimidine modified RNA with functional groups that is compatible with re-amplification, Nucleic Acids Res. 26(7): 1851-1853.

Kuwahara, M., Nagashima, J., Hasegawa, M., Tamura, T., Kitagata, R., Hanawa, K., Hososhima, S., Kasamatsu, T., Ozaki, H. \& Sawai, H. (2006). Systematic characterization of 2'-deoxynucleoside-5'-triphosphate analogs as substrates for DNA polymerases by polymerase chain reaction and kinetic studies on enzymatic production of modified DNA, Nucleic Acids Res. 34(19): 5383-5394.

Kuwahara, M., Obika, S., Nagashima, J., Ohta, Y., Suto, Y., Ozaki, H., Sawai, H. \& Imanishi, T. (2008). Systematic analysis of enzymatic DNA polymerization using oligo-DNA templates and triphosphate analogs involving 2',4'-bridged nucleosides, Nucleic Acids Res. 36(13): 4257-4265.

Kuwahara, M., Takeshima, H., Nagashima, J., Minezaki, S., Ozaki, H. \& Sawai, H. (2009). Transcription and reverse transcription of artificial nucleic acids involving 
backbone modification by template-directed DNA polymerase reactions, Bioorg. Med. Chem. 17(11): 3782-3788.

Lato, S. M., Ozerova, N. D. S., He, K., Sergueeva, Z., Shaw, B. R. \& Burke, D. H. (2002). Boron-containing aptamers to ATP. Nucleic Acids Res. 30(6): 1401-1407.

Lee, S. E., Sidorov, A., Gourlain, H., Mignet, N., Thorpe, S. J., Brazier, J. A., Dickman, M. J., Hornby, D. P., Grasby, J. A. \& Williams, D. M. (2001). Enhancing the catalytic repertoire of nucleic acids: a systematic study of linker length and rigidity, Nucleic Acids Res. 29(7): 1565-1573.

Lin, Y., Qiu, Q., Gill, S. C. \& Jayasena, S. D. (1994). Modified RNA sequence pools for in vitro selection. Nucleic Acids Res. 22(24): 5229-5234.

Mandal, M., Boese, B., Barrick, J. E., Winkler, W. C. \& Breaker, R. R. (2003). Riboswitches control fundamental biochemical pathways in Bacillus subtilis and other bacteria, Cell, 113(5): 577-586.

McNamara, J. O. II, Andrechek, E. R., Wang, Y., Viles, K. D., Rempel, R. E., Gilboa, E., Sullenger, B. A \& Giangrande, P. H. (2006). Cell type-specific delivery of siRNAs with aptamer-siRNA chimeras, Nat Biotechnol., 24(8): 1005-1015.

Mendonsa, S.D. \& Bowser, M. T. (2004). In vitro selection of high-affinity DNA ligands for human IgE using capillary electrophoresis, Anal. Chem., 76(18): 5387-5392.

Mendonsa, S. D. \& Bowser, M. T. (2005). In vitro selection of aptamers with affinity for neuropeptide Y using capillary electrophoresis, J. Am. Chem. Soc., 127(26): 93829383.

Mosing, R. K., Mendonsa, S. D. \& Bowser, M. T. (2005). Capillary electrophoresis-SELEX selection of aptamers with affinity for HIV-1 reverse transcriptase, Anal. Chem., 77(19): 6107-6112.

Nolte, A., Klussmann, S., Bald, R., Erdmann, V.A. \& Fürste, J. P. (1996). Mirror-design of Loligonucleotide ligands binding to ${ }_{\mathrm{L}}$-arginine, Nat. Biotechnol. 14(9): 1116-1119.

Pagratis, N. C., Bell, C., Chang, Y. F., Jennings, S., Fitzwater, T., Jellinek, D. \& Dang, C. (1997). Potent 2'-amino-, and 2'-fluoro-2'-deoxyribonucleotide RNA inhibitors of keratinocyte growth factor. Nat. Biotechnol, 15(1): 68-73.

Perrin, D. M., Garestier, T. \& Hélène, C. (2001). Bridging the gap between proteins and nucleic acids: a metal-independent RNAse A mimic with two protein-like functionalities, J. Am. Chem. Soc. 123(8): 1556-1563.

Raddatz, M. S., Dolf, A., Endl, E., Knolle, P., Famulok, M. \& Mayer, G. (2008). Enrichment of cell-targeting and population-specific aptamers by fluorescence-activated cell sorting, Angew. Chem. Int. Ed., 47(28): 5190-5193.

Robertson, D. L. \& Joyce, G. F. (1990). Selection in vitro of an RNA enzyme that specifically cleaves single-stranded DNA, Nature 344(6265): 467-468.

Ruckman, J., Green, L. S., Beeson, J., Waugh, S., Gillette, W. L., Henninger, D. D., ClaessonWelsh, L. \& Janjić, N. (1998). 2'-Fluoropyrimidine RNA-based aptamers to the 165amino acid form of vascular endothelial growth factor (VEGF165). Inhibition of receptor binding and VEGF-induced vascular permeability through interactions requiring the exon 7-encoded domain, J. Biol. Chem. 273(32): 20556-20567.

Sakthivel, K. \& Barbas, C. F. III. (1998). Expanding the potential of DNA for binding and catalysis: highly functionalized dUTP derivatives that are substrates for thermostable DNA polymerases, Angew. Chem. Int. Ed. 37(20): 2872-2875.

Sazani, P. L., Larralde, R. \& Szostak, J. W. (2004). A small aptamer with strong and specific recognition of the triphosphate of ATP, J. Am. Chem. Soc., 126(27): 8370-8371. 
Shangguan, D., Li, Y., Tang, Z., Cao, Z. C., Chen, H. W., Mallikaratchy, P., Sefah, K., Yang, C. J., Tan, W. (2006). Aptamers evolved from live cells as effective molecular probes for cancer study, Proc. Natl. Acad. Sci. U S A, 103(32): 11838-11843.

Shangguan, D., Meng, L., Cao, Z. C., Xiao, Z., Fang, X., Li, Y., Cardona, D., Witek, R. P., Liu, C. \& Tan, W. (2008). Identification of liver cancer-specific aptamers using whole live cells, Anal. Chem., 80(3): 721-728.

Shoji, A., Kuwahara, M., Ozaki, H. \& Sawai, H. (2007). Modified DNA aptamer that binds the $(R)$-isomer of a thalidomide derivative with high enantioselectivity, J. Am. Chem. Soc., 129(5): 1456-1464.

Sidorov, A. V., Grasby, J. A. \& Williams, D. M. (2004). Sequence-specific cleavage of RNA in the absence of divalent metal ions by a DNAzyme incorporating imidazolyl and amino functionalities. Nucleic Acids Res. 32(4): 1591-1601.

Smith, E. A., Thomas, W. D., Kiessling, L. L. \& Corn, R. M. (2003). Surface plasmon resonance imaging studies of protein-carbohydrate interactions, J. Am. Chem. Soc., 125(20): 6140-6148.

Smith, G.P \& Petrenko, V. A. (1997). Phage display, Chem. Rev. 97(2): 391-410.

Tasara, T., Angerer, B., Damond, M., Winter, H., Dorhofer, S., Hubscher, U. \& Amacker, M. (2003). Incorporation of reporter molecule-labeled nucleotides by DNA polymerases. II. High-density labeling of natural DNA, Nucleic Acids Res. 31(10): 2636-2646.

Tang, J., Xie, J., Shao, N. \& Yan, Y. (2006). The DNA aptamers that specifically recognize ricin toxin are selected by two in vitro selection methods, Electrophoresis, 27(7): 1303-1311.

Tetin, S. Y., Swift, K. N. \& Matayoshi, E. D. (2002). Measuring antibody affinity and performing immunoassay at the single molecule level, Anal. Biochem., 307(7): 84-91.

Tuerk, C. \& Gold, L. (1990). Systematic evolution of ligands by exponential enrichment: RNA ligands to bacteriophage T4 DNA polymerase., Science 249(4968): 505-510.

Uphoff, K. W., Bell, S. D. \& Ellington, A. D. (1996). In vitro selection of aptamers: the dearth of pure reason, Curr. Opin. Struct. Biol. 6(3): 281-288.

Vaish, N. K., Larralde, R., Fraley, A. W., Szostak, J. W. \& McLaughlin, L. W. (2003). A novel, modification-dependent ATP-binding aptamer selected from an RNA library incorporating a cationic functionality, Biochemistry, 42(29): 8842-8851.

Williams, K. P. \& Liu, X. -H. (1997). Schumacher, T. N. M., Lin, H. Y., Ausiello, D. A., Kim, P. S. \& Bartel., D. P. (1997). Bioactive and nuclease-resistant L-DNA ligand of vasopressin. Proc. Natl. Acad. Sci. USA, 94(21): 11285-11290.

Wilson, C., Nix, J. \& Szostak, J. (1998). Functional requirements for specific ligand recognition by a biotin-binding RNA pseudoknot, Biochemistry, 37(41): 14410-14419.

Winkler, W. C., Cohen-Chalamish, S. \& Breaker, R. R. (2002). An mRNA structure that controls gene expression by binding FMN, Proc. Natl. Acad. Sci. U S A., 99(25): 15908-15913.

Winkler, W. C., Nahvi, A. \& Breaker, R. R. (2002). Thiamine derivatives bind messenger RNAs directly to regulate bacterial gene expression, Nature 419(6910): 952-956.

Winkler, W. C., Nahvi, A., Sudarsan, N., Barrick, J. E. \& Breaker, R. R. (2003). An mRNA structure that controls gene expression by binding S-adenosylmethionine, Nat. Struct. Biol., 10(9): 701-707.

Zaug, A. J. \& Cech, T. R. (1986). The intervening sequence RNA of Tetrahymena is an enzyme, Science 231(4737): 470-475. 


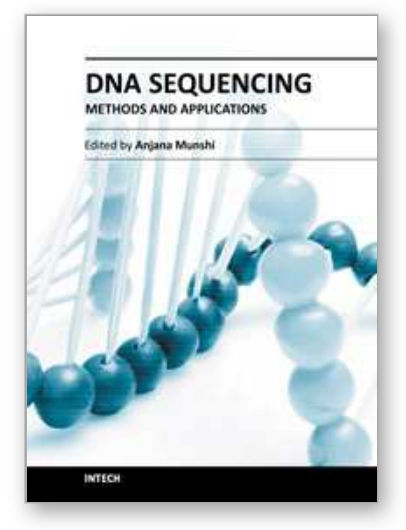

\section{DNA Sequencing - Methods and Applications}

Edited by Dr. Anjana Munshi

ISBN 978-953-51-0564-0

Hard cover, 174 pages

Publisher InTech

Published online 20, April, 2012

Published in print edition April, 2012

This book illustrates methods of DNA sequencing and its application in plant, animal and medical sciences. It has two distinct sections. The one includes 2 chapters devoted to the DNA sequencing methods and the second includes 6 chapters focusing on various applications of this technology. The content of the articles presented in the book is guided by the knowledge and experience of the contributing authors. This book is intended to serve as an important resource and review to the researchers in the field of DNA sequencing.

\section{How to reference}

In order to correctly reference this scholarly work, feel free to copy and paste the following:

Masayasu Kuwahara and Naoki Sugimoto (2012). Nucleic Acid Aptamers as Molecular Tags for Omics Analyses Involving Sequencing, DNA Sequencing - Methods and Applications, Dr. Anjana Munshi (Ed.), ISBN: 978-953-51-0564-0, InTech, Available from: http://www.intechopen.com/books/dna-sequencing-methods-andapplications/nucleic-acid-aptamers-as-molecular-tags-for-omics-analyses-involving-sequencing

\section{INTECH}

open science | open minds

\author{
InTech Europe \\ University Campus STeP Ri \\ Slavka Krautzeka 83/A \\ 51000 Rijeka, Croatia \\ Phone: +385 (51) 770447 \\ Fax: +385 (51) 686166 \\ www.intechopen.com
}

\author{
InTech China \\ Unit 405, Office Block, Hotel Equatorial Shanghai \\ No.65, Yan An Road (West), Shanghai, 200040, China \\ 中国上海市延安西路65号上海国际贵都大饭店办公楼 405 单元 \\ Phone: +86-21-62489820 \\ Fax: $+86-21-62489821$
}


(C) 2012 The Author(s). Licensee IntechOpen. This is an open access article distributed under the terms of the Creative Commons Attribution 3.0 License, which permits unrestricted use, distribution, and reproduction in any medium, provided the original work is properly cited. 